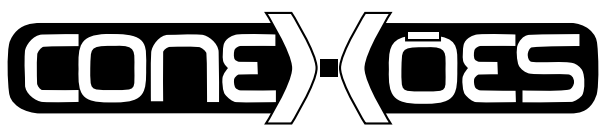

CIÊNCIA E TECNOLOGIA

\title{
VALIDAÇÃO DAS EQUAÇÕES ANALÍTICAS DE PLACA CIRCULAR CARREGADA AO LONGO DE UMA CIRCUNFERÊNCIA CONCÊNTRICA
}

\author{
ÍtAlo Linhares SAlomão ${ }^{1}$, EduARdo HÉlio Costa BARros ${ }^{1}$ \\ ${ }^{1}$ Universidade de Fortaleza (UNIFOR) \\ <italosalomao@hotmail.com>.<eduardohelio@hotmail.com>
}

DOI: 10.21439/conexoes.v13i3.1278

\begin{abstract}
Resumo. Dada a complexidade da análise de fundações circulares, o presente trabalho busca validar as equações analíticas de placa circular carregada ao longo de uma circunferência concêntrica com a mesma, a fim de viabilizar suas utilizações para a análise e dimensionamento de fundações circulares. Esta validação é realizada por meio de uma correlação entre deslocamentos e esforços internos de modelos numéricos bidimensionais e tridimensionais. A validação das equações foi alcançada através de comparativos entre resultados de dois modelos em elementos finitos, sendo o primeiro composto por elementos de placa espessa e o segundo por elementos tridimensionais tetraédricos, ambos carregados ao longo de uma circunferência com 4 metros de raio, concêntrica à fundação de 9 metros de raio. São apresentadas neste trabalho as características geométricas dos modelos, assim como as condições de apoio e de carregamento utilizadas. Os resultados obtidos de deslocamentos no sólido foram comparados com o modelo de placa e com as equações analíticas, tendo sido obtido erros de até $22 \%$, neste quesito. Para os momentos radiais, obteve-se discrepância de até $45 \%$. Por fim, para os momentos circunferenciais, obteve-se variação entre os modelos de $2 \%$ a $10 \%$. A partir destes resultados, para o caso em estudo, não é possível utilizar equações analíticas para o dimensionamento de estruturas de fundação circular devido aos seus valores terem sido demasiadamente diferentes dos modelos numéricos.
\end{abstract}

Palavras-chaves: Elementos finitos. Placas circulares. Fundação.

\section{VALIDATION OF ANALYTICAL EQUATION OF CIRCULAR PLATE LOADED ALONG A CONCENTRIC CIRCUMFERENCE}

\begin{abstract}
This paper seeks to validate the analytical equations of a circular plate loaded along a concentric circle with the plate, in order to facilitate their use for the analysis and design of circular foundations. This validation is accomplished by a correlation between displacements and internal forces of two-dimensional and three-dimensional models. The equations validation was achieved by the comparison between the results of two models in finite elements, the first being composed of thick-plate elements and the second of tetrahedral three-dimensional elements, both loaded along a circumference of 4 meters of radius, concentric with the 9 meters radius foundation. This study describe the geometric characteristics of the models, as well as the conditions of support and load that were used. The results of the displacements in the solid were compared with the ones in the plate and with the analytical equations, obtaining errors up to $22 \%$ in this regard. For radial moments, discrepancy was up to $45 \%$. Finally, for the circumferential moments, the obtained variation between models was of $2 \%$ to $10 \%$. From these results, for the case studied, it is not possible to use analytical equations for the design of circular foundation structures due to their values being too different of the numerical models.
\end{abstract}

Keywords: Finite elements. Circular plates. Foundation 


\section{INTRODUÇÃO}

Dada à complexidade da natureza, os sistemas físicos têm comportamentos muito complicados para uma perfeita compreensão matemática. Devido a execução rotineira de experimentos físicos para o entendimento aproximado destes comportamentos serem muito dispendiosos ou até mesmo impossível, são desenvolvidos métodos numéricos com essa mesma finalidade (SORIANO, 2009).

Dentre esses métodos, o mais utilizado devido a sua eficiência e sua vasta implementação em diversos programas computacionais é o Método dos Elementos Finitos ou MEF.

No âmbito da Engenharia de Estruturas, o MEF tem como objetivo a determinação do estado de tensão e deformação de um elemento de geometria arbitraria sujeito a ações externas. Ao projetar uma estrutura, é habitual proceder-se com uma sucessão de análises e simplificações com o objetivo de se alcançar uma solução satisfatória (AZEVEDO, 2015).

A fundamentação básica do MEF é que toda função contínua pode ser aproximada por um modelo composto de um conjunto de funções contínuas definidas sobre um número finito de subdomínios. A construção de um modelo discreto de um meio contínuo, ou domínio, consiste na divisão do mesmo em elementos, ou subdomínios, os quais são delimitados por um número finito de pontos. Esses pontos são chamados de nós ou pontos nodais. Cada subdomínio tem seu comportamento obtido através da integração do comportamento dos nós que o delimita, de modo a manter a continuidade ao longo das fronteiras entre os elementos.

Meios contínuos podem ser discretizados por elementos unidimensionais, bidimensionais e tridimensionais. A escolha de um modelo para a representação de uma estrutura depende da geometria de seus componentes, das ações externas e do comportamento que se deseja analisar. Quando apenas se considera uma dimensão, o método não apresenta grande interesse prático quando comparado com elementos de duas e três dimensões.

É sempre recomendado iniciar-se com um modelo simples e gradativamente sofisticá-lo, caso seja necessário. Na medida do possível, é melhor dar preferência a elementos bidimensionais em relação aos tridimensionais, e caso os unidimensionais se mostrem satisfatórios, dar preferência a esses elementos em relação aos bidimensionais (SORIANO, 2009).

Em detrimento da simplicidade sem a perda de eficácia, buscou-se a obtenção de resultados referentes a elementos tridimensionais e de placas, ambos circula- res, submetidas a flexão através de equações que simulam o comportamento de placas circulares delgadas também submetidas a flexão, ambos os casos apenas apoiados no bordo exterior.

\section{APRESENTAÇÃO DOS MODELOS}

A transposição de um modelo real para um modelo analítico e representativo consiste em idealizar simplificações plausíveis que sejam solucionáveis matematicamente e que sejam fidedignas à estrutura real.

Para o estudo de qualquer elemento estrutural, é necessário se determinar inicialmente um modelo validado e convergido, utilizado para a integração de resultados entre modelos numéricos, analíticos ou experimentais. No caso do presente trabalho, serão utilizados modelos numéricos aferidos entre si, e também baseados em equações analíticas.

Para a modelagem computacional, devem-se determinar os materiais, a geometria da estrutura, o tipo de elemento utilizado que descreva o comportamento do contínuo, as condições de contorno e as condições de carregamento.

O material utilizado apresenta as seguintes características: concreto com resistência característica a compressão de $35 \mathrm{MPa}$ (Fck), módulo de elasticidade secante de 28,160 GPa, coeficiente de Poisson de 0,2, módulo de elasticidade transversal de $11,733 \mathrm{GPa}$ e peso especifico de $25 \mathrm{kN} / \mathrm{m}^{3}$.

A geometria adotada para a fundação foi de 18 metros de diametro e 1 metro de altura, contabilizando um volume total de concreto de $254,47 \mathrm{~m}^{3}$ com aproximadamente $6361,7 \mathrm{kN}$.

Para representar a trasmissão dos esforços provenientes da superestrutura de maneira satisfatória e sem dissipação de energia, foi idealizado um sistema de barras e placas de tranferência para simular a ligação entre a fundação e a superestrutura. Tal sistema consiste na aplicação das ações em um ponto no centro de uma placa circular, com 4 metros de raio, concêntrica à fundação, estando a mesma ligada à fundação através de barras de transferência igualmente distribuídas em seu contorno. O material idealizado para o sistema de transferência possui a rigidez elevada, sendo esta no valor de 9 x $1015 \mathrm{MPa}$, para simular ligações rígidas.

Foram adotados dois modelos, o primeiro modelo de placa espessa com a espessura constante de 1 metro (Figura 1) a qual está de acordo com a teoria de Reissner-Mindlin para placas espessas, e o segundo modelo em elementos tridimensionais de espessura constante de 1 metro (Figura2). 


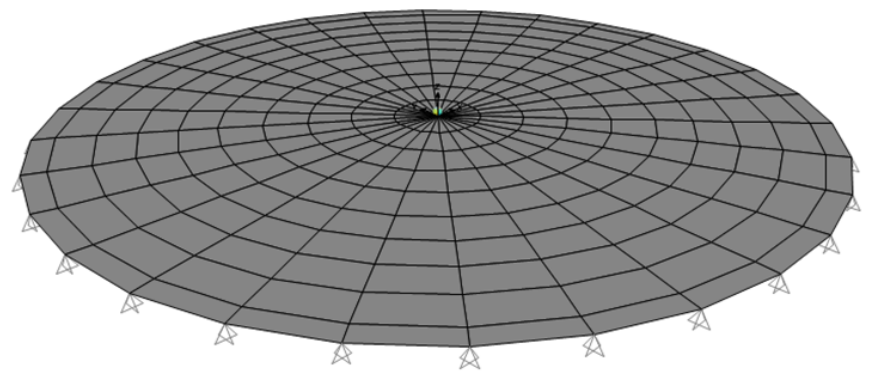

Figura 2: Modelo em Elementos Tridimensionais.

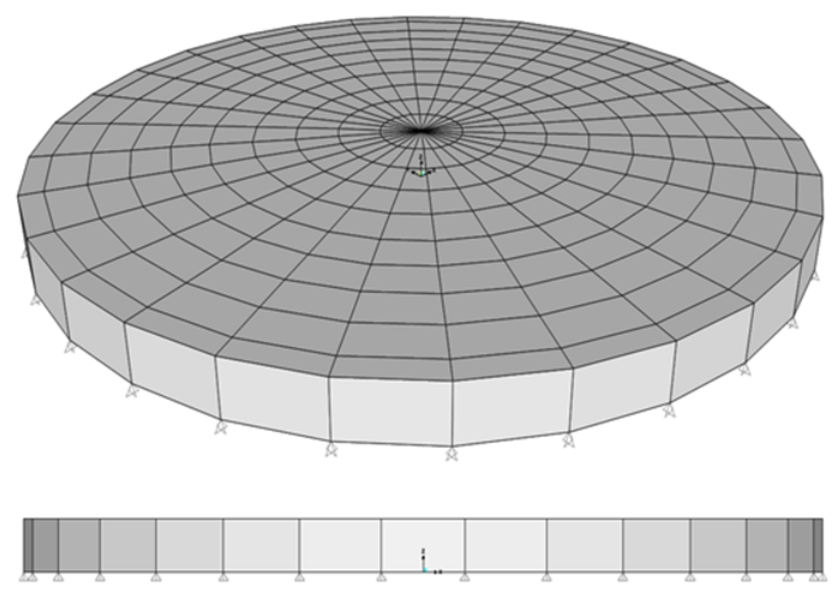

A discretização de uma malha de elementos finitos para simular placas circulares foi estudada por Barros, Genuino e Salomão (2015), cujos resultados das modelagens mostraram que, para a obtenção de um modelo válido de placas circulares, o que apresentou melhor convergência foi o com 8 graus de ângulo interno e 9 divisões equidistantes no sentido radial. Tal malha com 8 graus de ângulo interno representa 45 divisões no sentido circunferencial, cujo modelo foi utilizado no presente estudo, como mostram as Figuras 1 e 2 Ambos os modelos foram idealizados com o deslocamento de suas extremidade restrito verticalmente.

Os modelos em elementos tridimensionais podem ser considerados válidos, desde que sejam modelados nas dimensões corretas da peça e com as devidas condições de contorno e de carregamento. A análise de elementos finitos que possuem volume é pouco utilizada devido à dificuldade na geração da malha. Esta precisa ser, diversas vezes, totalmente manual, para que se tenha o controle de todos os nós. Há dificuldade também na interpretação dos resultados, pois esse modelo apresenta como solução apenas deslocamento e tensão, diferente dos outros elementos que tem como resultado o deslocamento e os esforços internos de momento e cortante.

O ideal, em termos de quantidade de dados analisados e resultados obtidos, é que consigamos um modelo parcimonioso, i.e., aquele que explica a maior quantidade de variância a partir do menor número de variáveis. Para se adotar um modelo como representativo, é necessário comparar diferenças de resultados de discretizações sucessivas em identificação de uma discretização que dispense refinamentos posteriores, ou seja, que não gere melhorias que justifiquem um aumento no número de variáveis.

Na etapa de Assembly, ou montagem do sistema, é necessário muita cautela, pois todos os passos influenciam no resultado final, desde a escolha dos tipos de elementos, como cascas, placas, sólidos dentre outros, até a conexão com exatidão dos pontos que delimitam os elementos para que a continuidade seja garantida, caso contrário, haverá um erro na transmissão dos esforços 
e na geração do campo de tensões naquela região, levando a uma interpretação correta de uma modelagem errada.

\subsection{Condições de carregamento}

O modelo de transmissão de ações utilizado baseiase na geometria da superestrutura que será acoplada no topo da fundação, para tal utilizou-se uma placa circular, no diâmetro da superestrutura, ligada ao bloco por pinos distribuídos igualmente espaçados em sua borda (Figura 3 ).

Foram utilizados elementos que simulam ligações rígidas a fim de transmitir os esforços para a fundação. Esse tipo de ligação tem a função de impedir a rotação relativa à flexão entre a placa, os pinos de transferência de esforços e o elemento de fundação, além de transmitir à peça de apoio a força cortante e a força normal.

Além do peso próprio da fundação no valor de $6361,7 \mathrm{kN}$, será considerada apenas uma carga vertical no topo da fundação, a favor da gravidade, no valor de 25000 kN. A ação e o peso próprio atuarão sem majoração, pois a intenção é comparar os resultados entre os dois modelos e não a determinação de uma situação limite para o dimensionamento da peça.

A verificação da transmissão é feita a partir da máxima força de compressão e de tração que surgem nos pinos ao serem aplicadas as cargas verticais e de flexão, podendo estas serem obtidas através das Equações 1 para máxima compressão, e 2, para máxima tração.

$$
\begin{aligned}
& F_{c}=\frac{N+\frac{2 M}{R}}{N_{\text {pinos }}} \\
& F_{t}=\frac{N-\frac{2 M}{R}}{N_{\text {pinos }}}
\end{aligned}
$$

Onde:

$\mathrm{N}$ é o esforço normal proveniente da superestrutura;

M é o momento de flexão proveniente da superestrutura;

$\mathrm{R}$ é o raio da placa de transferência;

$\mathrm{N}_{\text {pinos }}$ é o número de pinos que distribuirão a força na fundação.

\section{VALIDAÇÃO ANALÍTICA}

O cálculo analítico das placas circulares é possível, no caso de existir simetria das condições de contorno e das condições de solicitação em relação ao eixo normal à superfície média passando pelo centro da placa, sendo a integração analítica da equação de equilíbrio simples.
É conveniente utilizar um sistema de referência em coordenadas cilindricas, sendo a origem das coordenadas coincidente com o centro da placa antes da deformação (DINIS, 2016).

Para a situação em estudo podemos considerar que, através da placa e dos pinos de transferência, as cargas aplicadas no topo do sistema são transmitidas à fundação de maneira homogênea e atuam como uma carga linear situada na projeção do perimetro da placa de transferência.

Para a determinação do deslocamento transversal têm-se duas situações distintas, sendo a primeira quando a seção verificada estiver externa à seção de aplicação dos carregamentos, ou seja, quando $r>R_{i}$, podendo seu valor ser determinado através da Equação 3 A segunda hipótese é dada quando $r<R_{i}$, sendo o deslocamento transversal obtido pela Equação 4 Os momentos radiais e circunferenciais podem ser calculados a partir das Equações 5 e 6, sendo a integral do deslocamento em função da posição da seção a se determinar, i.e, para seções menores do que o raio de aplicação da carga, deve-se utilizar a integração da equação de deslocamento 3 caso contrário, utilizar a Equação 4 O valor do módulo de rigidez a flexão é representado pela letra D e calculado através da Equação 7

Onde:

P é a força vertical aplicada a placa;

$\mathrm{R}_{\mathrm{e}}$ é o raio externo;

$\mathrm{R}_{\mathrm{i}}$ é o raio de aplicação da força vertical; $\mathrm{r}$ é onde se deseja obter as resultantes de deslocamento e flexão;

v o coeficiente de Poisson.

A verificação dos momentos gerados pelo modelo tridimensional é feita a partir da transformação das tensões obtidas. Essa transformação é feita multiplicandose a tensão obtida pelo módulo resistente na seção desejada a cada metro de comprimento.

\section{RESULTADOS E DISCUSSÕES}

A aferição dos resultados de deslocamentos foi feita em relação aos resultados analíticos e para ambos os modelos. Tais deslocamentos foram obtidos em diversas seções com raios conforme apresentado na Tabela 1 e representados no Gráfico 1

Como o método dos elementos finitos tem como variável principal o deslocamento nodal, sendo realizado, a partir destes deslocamentos, as integrações para que se obtenham resultantes de tensão e de momento, é conveninente a verificação inicial da convergência de valores de deslocamento para, posteriormente, verificar os 


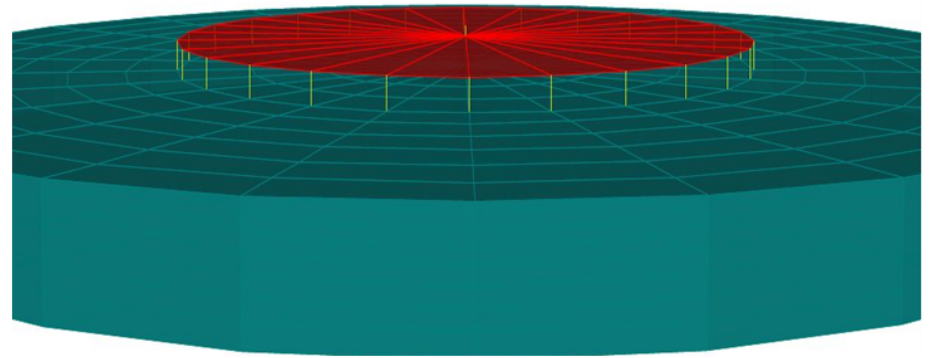

$$
\begin{gathered}
\omega=\frac{P}{8 \pi D}\left(R_{e}{ }^{2}+r^{2} \hat{M}^{\bigotimes} 1+\frac{1}{2} \frac{(1-v)}{(1+v)} \frac{\left(R_{e}^{2}-R_{i}^{2}\right.}{R_{e}}+\left(R_{e}^{2}-r^{2}{ }^{\hat{M}} \ln \frac{r}{R_{e}}\right.\right. \\
\omega=\frac{P}{8 \pi D}\left(R_{i}{ }^{2}+r^{2} \hat{M}^{\hat{M}} \frac{R_{i}}{R_{e}}+\left(R_{e}{ }^{2}+R_{i}{ }^{2} \frac{\hat{M}(3-v) R_{e}{ }^{2}-(1-v) r^{2}}{2(1+v) R_{e}{ }^{2}}\right.\right.
\end{gathered}
$$

$$
\begin{gathered}
M_{r}=-D^{\otimes} \frac{d^{2} \omega}{d r^{2}}+\frac{v}{r} \frac{d \omega^{\bigotimes}}{d r} \\
M_{\theta}=-D \frac{1}{r} \frac{d \omega}{d r}+v \frac{d^{2} \omega^{\otimes}}{d r^{2}} \\
D=\frac{E e^{2}}{12\left(1-v^{2}\right)}
\end{gathered}
$$




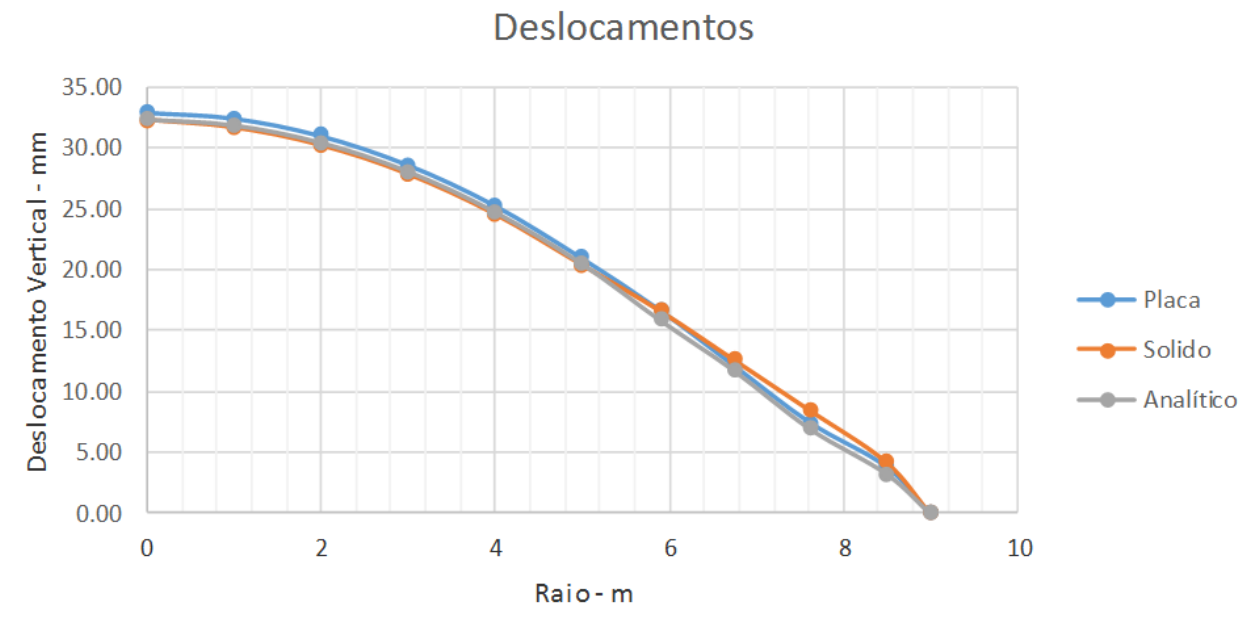

Tabela 2: Tensões e momentos resultantes no sólido

\begin{tabular}{c|c|c|c|c}
\hline \multicolumn{5}{c}{ Sólido } \\
\hline Raio da Seção (m) & $\sigma_{11}\left(\mathbf{N} / \mathbf{m m}^{2}\right)$ & $\mathbf{M}_{11}(\mathbf{k N . m})$ & $\sigma_{22}\left(\mathbf{N} / \mathbf{m m}^{2}\right)$ & $\mathbf{M}_{22}(\mathbf{k N . m})$ \\
\hline 1,00 & 15,29 & 2548,28 & 15,81 & 2635,00 \\
\hline 2,00 & 15,29 & 2548,98 & 15,22 & 2536,67 \\
\hline 3,00 & 15,35 & 2558,33 & 15,34 & 2556,67 \\
\hline 4,00 & 15,30 & 2550,00 & 15,22 & 2536,67 \\
\hline 5,00 & 15,24 & 2540,00 & 15,26 & 2543,33 \\
\hline 5,90 & 10,88 & 1813,33 & 13,80 & 2300,00 \\
\hline 6,75 & 7,69 & 21281,67 & 12,46 & 2076,67 \\
\hline 7,63 & 3,05 & 508,33 & 9,84 & 1640,00 \\
\hline 8,50 & 1,55 & 258,33 & 8,60 & 1433,33 \\
\hline 9,00 & 0,67 & 111,67 & 7,77 & 1295,00 \\
\hline \multicolumn{5}{|c|}{}
\end{tabular}

Figura 4: Direções Principais

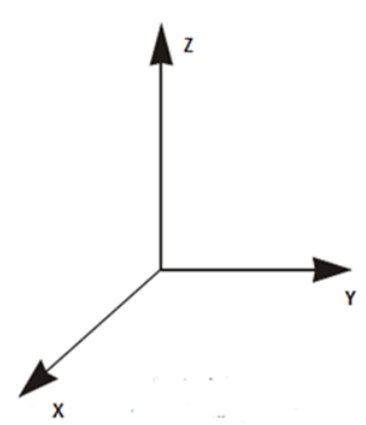

Os Gráficos 2 e 3 nos mostram a relação entre os momentos obtidos através das tensões resultantes do modelo tridimensional, dos momentos obtidos no modelo de placas e através das equações analíticas.

O estudo apresentado é delimitado pelas Equações

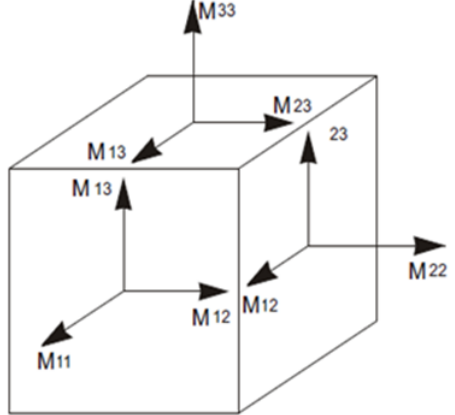

$3 \mathrm{a} 7$, sendo estas validas apenas para placas circulares simplesmente apoiadas com carregamento concêntrico. A partir desta imposição literária, buscou-se um modelo numérico que delibera-se resultados representativos. Deste modo, foram criados elementos circulares, 
Tabela 3: Momentos na placa.

\begin{tabular}{c|c|c}
\hline \multicolumn{3}{|c}{ Placa } \\
\hline Raio da Seção (m) & $\mathbf{M}_{11}(\mathbf{k N . m})$ & $\mathbf{M}_{22}(\mathbf{k N . m )}$ \\
\hline 1,00 & 2592,82 & 2592,82 \\
\hline 2,00 & 2592,82 & 2592,82 \\
\hline 3,00 & 2592,82 & 2592,82 \\
\hline 4,00 & 2592,82 & 2592,82 \\
\hline 5,00 & 2592,82 & 2578,96 \\
\hline 5,90 & 1752,32 & 2321,1 \\
\hline 6,75 & 1221,68 & 2021,36 \\
\hline 7,63 & 450,99 & 1612,96 \\
\hline 8,50 & 140,22 & 1406,29 \\
\hline 9,00 & 99,63 & 1290,67 \\
\hline
\end{tabular}

Tabela 4: Momentos analíticos.

\begin{tabular}{c|c|c}
\hline \multicolumn{3}{c}{ Analítico } \\
\hline Raio da Seção (m) & $\mathbf{M}_{11}(\mathbf{k N . m})$ & $\mathbf{M}_{22}$ (kN.m) \\
\hline 1,00 & 2574,54 & 2574,54 \\
\hline 2,00 & 2574,54 & 2574,54 \\
\hline 3,00 & 2574,54 & 2574,54 \\
\hline 4,00 & 2574,54 & 2574,54 \\
\hline 5,00 & 1755,34 & 2328,30 \\
\hline 5,90 & 1216,68 & 2076,69 \\
\hline 6,75 & 809,05 & 1841,70 \\
\hline 7,63 & 455,75 & 1609,89 \\
\hline 8,50 & 155,49 & 1394,59 \\
\hline 9,00 & 0,00 & 1277,17 \\
\hline
\end{tabular}

Grafico 2: Momentos radiais (M11) nos modelos solido e de placa.

Momentos Raiais - M11

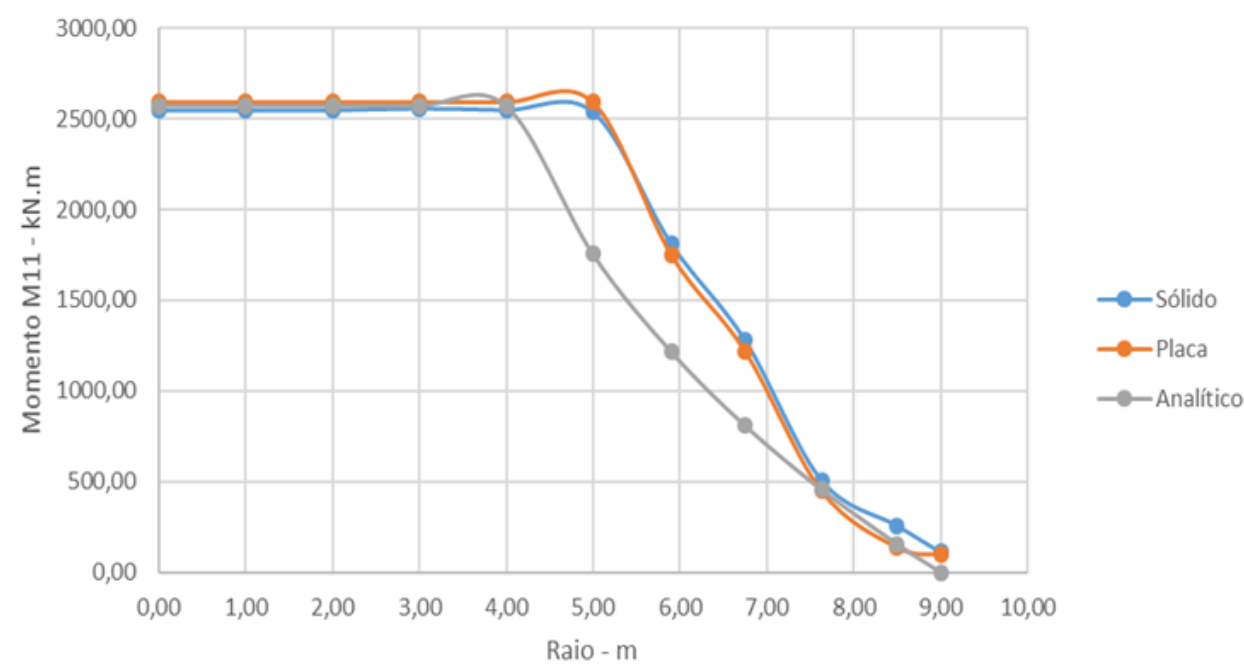

sejam planos ou espaciais, que tivessem restrições de apoio translacionais, livres a rotações, e a aplicação do carregamento através de elementos rígidos, transpondo as cargas lineares em nodais.
É importante ressaltar que as equações apresentadas na literatura não delimitam as suas condições de uso, no entanto, ao realizar uma sobreposição dos gráficos 1 a 3 . é notória a fronteira no que se refere à precisão de 


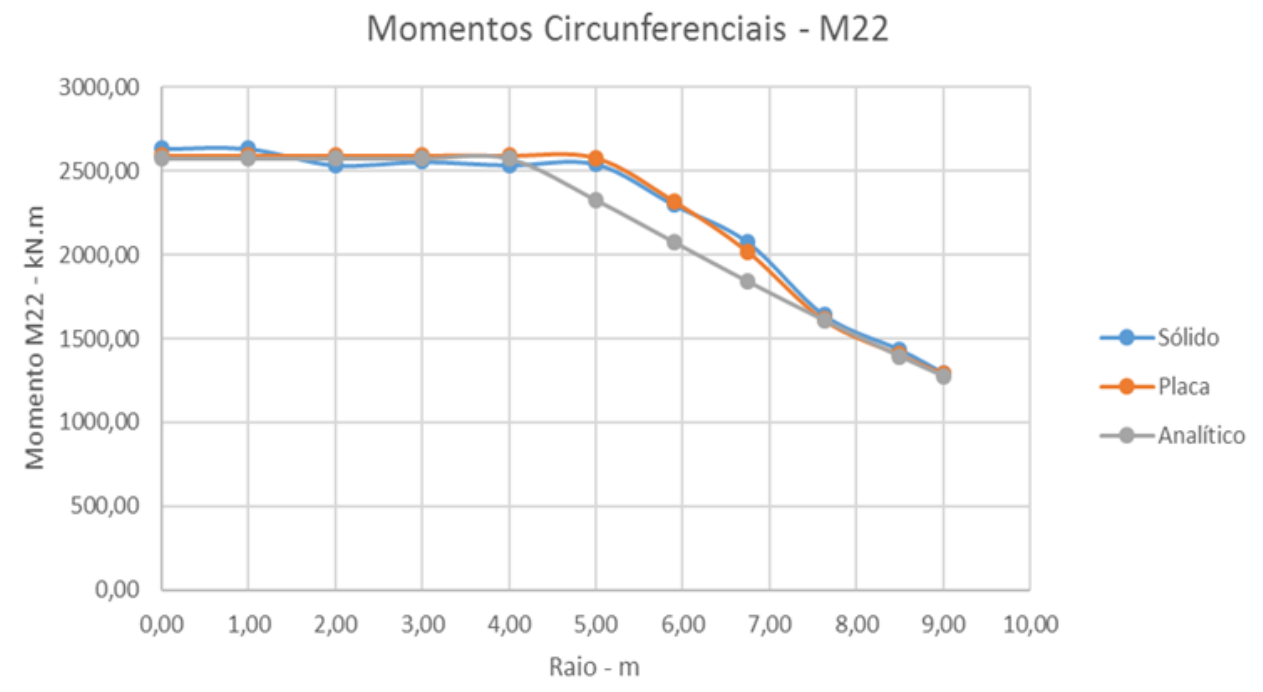

valores quanto ao tamanho da base, limitado a 4 metros os resultados determinados analiticamente que se aproximam dos obtidos nos modelos numéricos. Deve-se, portanto, avaliar se o grau de precisão a qual a estrutura a ser calculada necessita.

\section{CONCLUSÕES}

A fim de validar as equações analíticas para o cálculo dos deslocamentos, assim como dos momentos radiais e circunferenciais de placas circulares, tendo como principal objetivo a análise de fundações circulares, foi realizado o estudo de uma base através do método dos elementos finitos, sendo seus resultados comparados com os resultados analíticos.

Podemos constatar que, para os deslocamentos, houve uma variação média de aproximadamente $5 \%$ entre os modelos analíticos e numéricos. Obteve-se uma maior divergência entre os valores nos raios de 7,63 e 8,5 metros, apresentando valores de $7 \%$ para o comparativo entre o modelo analítico e o de placa, e no valor máximo de $22 \%$ entre o modelo analítico e sólido. No restante das seções, a variação entre deslocamentos foi de 0,6 a $6,8 \%$. Tem-se, portanto, que, tomando como base o modelo sólido, é possível a verificação dos deslocamentos da base para um raio de até aproximadamente 6 metros, não sendo acurado avaliar os deslocamentos por processos simplificados no restante da base.

Para os momentos radiais, foram observadas duas situações distintas, sendo a primeira os momentos medidos com o raio menor do que o raio de aplicação da carga. Neste caso os valores variaram de $0,7 \%$ até $1,7 \%$ entre todos os modelos. Para a situação com o raio maior do que o raio de aplicação da força, a diferença entre os modelos foi considerável, apresentando valores de até $45 \%$, invalidando, assim, as equações analíticas para a determinação dos momentos radiais.

Por fim, os momentos circunferenciais apresentaram, em todas as seções, erros variando em torno de $2 \%$, tendo seu valor extremo de $10 \%$, sendo este aceito por tratar de métodos aproximados de solução de modelos contínuos.

Devido às fundações se tratarem de estruturas com alto grau de precisão, a utilização das equações analíticas para determinação do deslocamento e dos esforços internos mostraram-se, para o caso estudado, inadequadas.

\section{AGRADECIMENTOS}

Os autores agradecem ao Programa de Iniciação Cientifica da Fundação Edson Queiroz e ao Departamento de Engenharia Civil da Universidade de Fortaleza (UNIFOR) pelo apoio na realização desta pesquisa.

\section{REFERÊNCIAS}

AZEVEDO, A. F. M. Método dos Elementos Finitos. 2003. Disponível em: <http://www.alvaroazevedo. com/publications/books/livro_mef_aa_1ed/>. Acesso em: 13 maio 2016. 
BARROS, E. H. C.; GENUINO, V. d. J.; SALOMÃO,

I. L. Estudo da convergência e validação de um modelo de placa circular em elementos finitos. In: ENCONTRO DE INICIAÇÃO À PESQUISA DA

UNIFOR, 11., 2015. Fortaleza, 2015.

DINIS, L. M. d. J. S. Placas e Cascas:

Capítulo 5. 2004. Disponível em: <https:

//web.fe.up.pt/ ldinis/cap5placas.pdf > Acesso em: 13 maio 2016.

SORIANO, H. L. Elementos Finitos: Formulação e Aplicação na Estática e Dinâmica das Estruturas.

Rio de Janeiro: Ciência Moderna, 2009. 